\title{
Die Kybernetik hört nicht auf
}

Max Stadler

Julian Bauer 2016: Zellen, Wellen, Systeme. Eine Genealogie systemischen Denkens, 1880-1980. Historische Wissensforschung 5. Tübingen: Mohr Siebeck, 360 S., € 49,00, ISBN 978-3-16-154679-2.

Hunter Heyck 2015: Age of System: Understanding the Development of Modern Social Science. Baltimore: Johns Hopkins Press, 272 p., \$ 54,95, ISBN: 978-1-42-141710-3.

Jan Müggenburg 2018: Lebhafte Artefakte. Heinz von Foerster und die Maschinen des Biological Computer Laboratory. Konstanz: Konstanz University Press, 380 S., € 39,90, ISBN: 978-3-83539103-1.

What you're trying to crack is the code of my dance. Heinz von Foerster, April 1974

Ebenso wie die frühen Kybernetiker bin ich stets zum aktiven Tanz im Dia$\log$ bereit und hätte Sie diesbezüglich am 9. April morgens auf dem ICEBahnsteig in Düsseldorfansprechen können. Offener Brief an Richard David Precht, April 2018

Hätte. Anlass des gescheiterten Dialogs waren mehrere, offenbar etwas unbedacht geäußerte Verlautbarungen Prechts - im Focus, im Spiegel und im ZDF - zur Sache der Digitalisierung. Namentlich die Diffamierung: „Das Menschenbild des Silicon Valley ist das der Kybernetik, nicht das der Aufklärung" sorgte in der Blogosphäre für Unmut. Denn, so ließ es sich im postwendend nachgereichten, offenen Brief an Richard David Precht nachlesen, „frühe Kybernetiker“ wie Norbert Wiener, Heinz von Foerster oder Warren McCulloch waren (wie Philosophen dies eigentlich wissen sollten) ausgemachte Humanisten, wenn nicht sogar „Anarchisten und Hyperkrea- 
tive“; mit den Machenschaften von Google, amazon oder facebook, mit kalifornischer Ideologie und unaufgeklärten Menschenbildern, hat die eigentliche Kybernetik jedenfalls nichts gemein (Paul 2018).

Wie man in dieser Sache - Digitalisierung, das Erbe der Kybernetik, die Algorithmen usw. - also auch steht, eine gewisse Aktualität der Thematik, um die auch die Bücher kreisen, die im Folgenden zur Diskussion stehen, wird man nicht abstreiten wollen. Um Kybernetik und Systemlogiken dreht es sich nämlich auch dort. Und zweitens dreht es sich um die Frage, welche Geschichte man eigentlich erzählen will, erzählt man davon. Vom Kalten Krieg? Von Cyborgs und Maschinenwerdung? Von den Vordenkern postindustrieller Zustände? Um dies schon einmal vorwegzunehmen: Gängige bzw. eindeutige Antworten aus der Rubrik Humanismus ja/nein finden sich bei den drei hier zu Wort kommenden Autoren nicht. Die Frontverläufe der einschlägigen Historiographie spiegelt der obige Zwischenfall am ICEBahnsteig in Düsseldorf aber immerhin ganz gut. Auf der einen Seite wäre da also die Geschichte, die auch Precht beim Nachdenken über das Silicon Valley im Hinterkopf gehabt haben mag (kürzlich wieder nachzulesen etwa in Thomas Rids Maschinendämmerung (2016)): die Kybernetik als Kriegsprodukt und Technokratenwissenschaft - ein Nimbus, der sie, aus guten Gründen, eigentlich immer schon umweht; endgültig eingeschliffen aber hatte sich dieser eher zweifelhafte Ruf in Zeiten von Reagan, PershingRaketen und Star Wars. ${ }^{1}$ Und auf der anderen Seite, eben der humaneren, da wäre die Kybernetik als mehr oder weniger undiszipliniertes, para-akademisches Weltanschauungs-Programm, dem Sozialwissenschaftler und Hirnforscher fast ebenso viel abgewinnen konnten wie Familientherapeuten, Büroplaner oder Aussteigerkommunen. ${ }^{2}$

Wissenschafts- und technikhistorisch betrachtet kommt der Kalte Krieg mittlerweile zwar nicht mehr ganz so kalt daher - und in jedem Fall unübersichtlicher -, als das Titel wie The Closed World (Edwards 1988) oder The Ontology of the Enemy (Galison 1994) noch nahelegten; und die Hippies geraten heute schnell mal in Verdacht, höchstselbst die kalifornische Ideologie miterfunden zu haben. ${ }^{3}$ Was die Kybernetik im engeren Sinne betrifft, blieb der basic plot allerdings erstaunlich stabil. Die Dramaturgie der Ereignisse ist insofern bekannt: Erfindung im Zweiten Weltkrieg in den USA (wobei es seit jeher ein beliebter Sport war, nach anderen, deutschen, britischen usw. proto-Kybernetikern $\mathrm{zu}$ fahnden), gefolgt von Blütephase, Niedergang, und einer Art Nachbeben in den 1970er Jahren, die „Kybernetik zweiter Ordnung“, deren Vertreter dann allerdings auch nur eingefleischteren Kennern ein Begriff sein dürfte. Korrekter wäre es wohl ohnehin von Nachleben im Plural zu sprechen. Denn so schwer man sich in der Gesamtschau auch tut, einzugrenzen, was diese Kybernetik nun eigentlich war - Universalwissenschaft, Technokraten-Ideologie, Metaphy- 
sik oder vielleicht doch etwas ganz Anderes -, umso einfacher ist es in der Regel, über ihre ideengeschichtlichen Ausläufer zu stolpern. Ob man dazu gleich das ganze Informationszeitalter rechnen muss, wie Ronald Kline dies unlängst in The Cybernetics Moment: Or Why We Call Our Age the Information Age nahegelegt hat, sei hier dahingestellt (Kline 2015). Nach wie vor aber - apropos Digitalisierung - geht von der versandeten Theorie-Unternehmung eine nicht zu bestreitende Strahlkraft aus. Soeben erst wurde etwa in Berlin die "Aktualisierung kybernetischen Denkens“ eingefordert (die Welt, hieß es da, sei „unwissentlich durchkybernetisiert") - inklusive tradition building in puncto "Regeltechnik“ aus deutschen Landen.

In Zeiten von Industrie 4.0, Automationsangst und nationaler AI-Strategien ist der Verweis auf die Pionierleistungen der Kybernetik jedenfalls selten weit - ohne aber, so steht zu vermuten, schon immer zum Verständnis derartiger Zustände beizutragen. Geschichten der (digitalen) Gegenwart kommen heute jedenfalls auch ganz gut ohne kybernetische Meisterdenker aus ${ }^{5}$ - ein Umstand, der eigentlich auf der Hand liegt, denn nicht nur gilt die Kybernetik gemeinhin als gescheiterte Bewegung; der Weg von den Macy Conferences hin zum, sagen wir, real existierenden Heimcomputer (geschweige denn, hin zu den Arbeitsbedingungen in einer Halbleiterfabrik irgendwo in Südostasien) war unter fast jeder Voraussetzung denkbar weit.

Aber selbst im engeren Referenzrahmen der hier interessierenden Wissens- bzw. Ideengeschichte kybernetisch-systemischen Denkens verblassen Chiffren wie Wiener, Shannon, oder von Neumann zusehends. Hunter Heycks Age of System. Understanding the Development of Modern Social Science - eines der drei hier besprochenen Bücher - etwa zeigt eindrücklich, wie sich diese und ähnliche cyber-historiographischen Allgemeinplätze mehr oder weniger systematisch umschiffen lassen (dazu unten also mehr). Was umgekehrt nicht heißen soll, die großen Denker*innen des Systems wären damit vernachlässigbar. Denn über deren Walten und Wirken ist das letzte Wort wohl ebenfalls noch nicht gesagt (auch dazu unten mehr). Um eine Diagnose Valentin Groebners etwas umzumünzen, könnte man in der Hinsicht sogar geneigt sein zu sagen: Die Kybernetik, darin dem Mittelalter nicht unähnlich, scheint gar nicht aufhören zu wollen (Groebner 2008). Allein, um beim obigen Beispiel zu bleiben, in besagten deutschen Landen findet sich bei näherer Betrachtung nicht so sehr „Niedergang“ als eine mehr oder weniger ununterbrochene Kette von Reanimationsversuchen, Anleihen und Instrumentalisierungen. Das reicht von den Auseinandersetzungen der 1960er Jahre im Stil der Two Cultures-Kontroverse, die insbesondere im Dreieck Ulm-Stuttgart-Karlsruhe kultiviert wurden (z.B. Steinbuch 1969), über die ökologisch angehauchte Konjunktur des „vernetzten Denkens“ in den 1970er Jahren (z. B. Vester 1978), hin zu den 
Neu- und Umentdeckungen um 1980, die dazumal Geisteswissenschaftler von Freiburg bis Bielefeld, und von Siegen bis West-Berlin in Beschlag nahm (z. B. Hombach 1982). Nicht zuletzt die 1990er und frühen 2000er Jahre jedoch machten die Kybernetik kulturwissenschaftlich hoffähig, als man sich von de:bug (Heidenreich \& Krajewski 2001) bis zur FAZ (Kittler 1994) über die Kybernetik informieren konnte (und neu: im „cyberspace“).

\section{Kybernetik à la viennoise}

Über derartige Genealogien kybernetischen Denkens weiß man gar nicht so viel. Umso erfreulicher ist es, dass mit Jan Müggenburgs Lebhafte Artefakte. Heinz von Foerster und die Maschinen des Biological Computer Laboratory nun eine substanzielle Studie zu einem jener „frühen Kybernetiker“ vorliegt, der in den späten 1970er und 1980er Jahren wie kaum ein Zweiter zum Stichwortgeber solchen Denkens avancierte: Heinz von Foerster (1911-2002). Letzteres - Foersters Pensionierungsphase sozusagen, als dieser zwischen Seewiesen, Big Sur und Münchner Kreis als Handlungsreisender in Sachen radikaler Konstruktivismus Karriere machen sollte - wird dabei zwar nur am Rande gestreift. ${ }^{6}$ Müggenburgs Hauptaugenmerk gilt Foersters, wenn man so will, aktiver Schaffensphase. Doch sie gilt damit dem Ort, an dem Foersters zunehmend häretische Thesen - „objectivity“ sei eine „peculiar delusion within our Western tradition“, so missionierte der österreichische Expat in späteren Jahren gerne (Foerster 1979) - überhaupt erst zusammengebastelt wurden: das Biological Computing Laboratory an der University of Illinois at Urbana-Champaign (UoI), kurz BCL.

Über jenes BCL konnte man sich bis dato vor allem in diversen Festschriften informieren (Müller \& Müller 2007). Lebhafte Artefakte nähert sich dem charmanten Wiener nun mit dem distanzierteren Blick des Wissens- und Medienhistorikers, um sich, so heißt es im Untertitel, den Maschinen des 1958 aus der Taufe gehobenen BCL anzunehmen. Naheliegenderweise natürlich, gehörte es doch zum Kerngeschäft der Kybernetik, eben diese zu ersinnen - und zwar üblicherweise solche, die einen gewissen Showwert besaßen (man denke etwa an Grey Walters Roboterschildkröten Elmer und Elsie). Die Vignette aus dem Jahr 1965, die Müggenburgs Buch vorangestellt ist, bündelt diese gerne übergangene Dimension kybernetischen Tuns gewissermaßen symptomatisch: Sie zeigt von Foerster im New Yorker Fernsehstudio der CBS bei der Vorführung seiner „Numarete“, einer delikaten Mustererkennungs-Apparatur, der sich nur mit einiger Trickserei der Anschein intelligenten Verhaltens einhauchen ließ. 
„Lebhafte Artefakte“ lautet das entsprechende Konzept, mit dem sich Müggenburg im Fortgang Foersters gekonntem Manövrieren zwischen Theoriebildung und Vorführkunst annähert. Gleich mehrere Dinge sind damit angesprochen. Zum einen: der Ort des BCL in der US-amerikanischen Wissenschaftslandschaft der Nachkriegsjahrzehnte, deren Strukturen dazu tendierten, Forscher*innen möglichst tangible Erzeugnisse abzuverlangen. Zum anderen: die epistemische Karriere des Modell-Begriffs selbst, um den sich die Kybernetik nachhaltig verdient gemacht hat; auch insofern sie, so Müggenburg, bestimmte Phänomene des Lebendigen überhaupt erst als in maschinenüberführbare zurechtgelegt hat (also etwa: Zählen, Mustererkennung, Informationsspeicherung, S. 328). Die biologisch anmutende Hardware, der man sich am BCL verschreiben sollte, ähnelt damit einer Art Vexierbild: „Erkenntniswerkzeuge“ einerseits; auf den „ästhetische[n] Effekt eines Anscheins von Belebtheit" getrimmte Illusionsapparate andererseits, die nicht zuletzt die (zumeist) militärischen Geldgeber einzunehmen hatten (S. 20-21). In Müggenburgs MaschinenBuch gerinnt dieser Überschuss zur These darüber, wie am BCL die Kybernetik in eine Kybernetik zweiter Ordnung mutierte - nämlich deren Kreisen um die "Selbstreflexivität“.

Entfaltet werden diese Überlegungen chronologisch: Kapitel 1 (von insgesamt vier) begleitet den einstigen Kybernetik-Guru auf den Weg in die USA - von dessen Trockenübungen als jugendlicher Zauberkünstler im Wien der 1920er und 1930er Jahre, über Foersters Kriegsjahre im Dienst der Hochfrequenzforschung und ein Nachkriegsintermezzo beim Radiosender Rot-Weiss-Rot. Der Virtuoso „persuasiver Demonstrationswerkzeuge" ist hier sichtlich schon angelegt, auch wenn es nicht unbedingt Vertrauen in Foersters epistemic virtues weckt, wenn man etwa erfährt, dass dessen erste biophysikalischen Gehversuche - eine quantenphysikalische Abhandlung zum "Gedächtnis“ (1948) - in zwei schlaflosen Nächten unter Hilfestellung einer "Schachtel Benzidrin" entstanden (S. 49). Wie dem auch sei: Über Umwege geriet diese Schrift in die Hände des US-amerikanischen Neuropsychiaters Warren McCulloch - und Foerster ans Department for Electrical Engineering der UoI. Zur Gründung des BCL als „verspätetes Labor" (S. 35) - eines von gerade mal zwei Laboren auf US-amerikanischen Boden, das ,Kybernetik' sich so auch auf die Fahnen schrieb -, sollte es allerdings noch etwa 10 Jahre dauern. Während nämlich der Stern der Kybernetik allmählich sank und namhafte Forscher sich distanzierten, vegetierte auch Foersters Karriere als Elektroingenieur dahin - „Von Foerster never saw it [solid-state physics] coming", sollte ein ehemaliges BCL-Mitglied sich diesbezüglich erinnern: „Didn’t see the future“ (S. 69).

Doch ein Leben jenseits der Normalwissenschaft war möglich, wie Müggenburgs Rekonstruktion des Ideenfelds, in welches sich das BCL nach 
1958 einnisten sollte, zeigt. Insbesondere betraf das „systems of ,quasibiological' complexity" (S. 141) - ein Gegenstand, der den Sorgen, Wünschen und Bedürfnissen des damals größten Kunden elektronischer Dinge überhaupt, dem US-Militär, sichtlich entgegenkam. (Eine B-52 etwa bestand 1955 aus immerhin 50.000 elektronischen Bauteilen (S. 287), was entsprechende „reliability“-Bedenken nach sich zog.) ${ }^{7}$ Müggenburgs Analyse der stets prekären Finanzierungsverhältnisse der von Foerster'schen Unternehmung verfährt detailliert und mehrschichtig. Man erfährt dabei nicht wenig über die diversen (überwiegend) militärischen Finanziers, die das BCL mal in diese, mal in jene Richtung schubsten; und vieles über die wissensgeschichtlichen Horizonte derart spekulativen Maschinenbaus. Auch die These, dass sich auf diesem Weg eine Art epistemischer Druck in Richtung Kybernetik zweiter Ordnung aufbaute, wird so plausibel. Denn letztere - „a cybernetics of observing systems rather than of observed systems" (Foerster) - sollte sich unter anderem dadurch auszeichnen, dem „Beobachter" solcher Vorführungen einen zentralen Stellenwert zuzuweisen - ein Trend hin zur Theorie, der sich verstärkte, als das BCL Ende der 1960er Jahre zunehmend zum Refugium alternder Kybernetiker und neuer Alliierter aufstieg: darunter der Hegel-versierte Cyberphilosoph Gotthard Guenther, der Computermusiker Herbert Brün und der chilenische Neurobiologe Humberto Maturana. Ferner war dies ein Trend, der sich verstärkte, als sich - im Gefolge von Vietnam, „Great Society“-Programm, Ende des Wachstums usw. - der Wind in Sachen Forschungsförderung spürbar drehte.

Foerster, mindestens ebenso adaptiv wie die Systeme, die er studierte, sattelte also einmal mehr um: Seminare zu „Ökologie“, „Selbsterfahrung" und „Drogenkonsum“ gehörten schon bald zum Portfolio des BCL (S. 324). Ein bisschen schade ist, dass dieser weitere Verlauf der Geschichte - Foersters späte Karriere als gegenkultureller Botschafter von Autopoiesis, Autonomie und Selbstreflexivität - nur noch am Rande verhandelt wird, auch wenn es wohl ein anderes, weniger maschinenlastiges Buch geworden wäre. Zu vermuten ist, dass die Kybernetik Foerster'scher Machart so weniger als (rein) epistemologisch-maschinelles als vielmehr als weltanschaulich-ideologisch motiviertes Programm dahergekommen wäre. Des Meisters Anleihen bei den Teachings of Don Juan, seine Auftritte in Esalen oder die Invektiven gegen die westliche Objektivitätsbesessenheit (,a contagious delusion") sprechen dafür. Schade ist dies nicht zuletzt, weil das ideelle Strandgut des BCL gegen Mitte/Ende der 1970er Jahre auch in der BRD angespült werden sollte - mit handgreiflichen Konsequenzen (wie Müggenburg andeutet). In Bremen sorgte sich etwa der junge Hirnforscher Gerhard Roth darum (im Rahmen des Forschungsschwerpunkts „Stabilitätsgrenzen biologischer Systeme“); in Siegen die Medienwissen- 
schaftler-in-the-making (LUMIS); und in Bielefeld die Sozialwissenschaft à la Luhmann. Hier liegt, anders gesagt, wohl ein interessantes Stück BRDGeistesgeschichte vergraben: irgendwo zwischen anschwellendem Mediendiskurs und neurobiologischem Naturalismus, zwischen UnregierbarkeitsKopfschmerzen, Geistesaustreibung (aus den Geisteswissenschaften) und New Ageism.

\section{Systemzeit}

Könnte man im Falle Foersters noch dazu geneigt sein, derartige Werdegänge als das idiosynkratische Treiben von „Anarchisten und Hyperkreative[n]“ zu entschuldigen, legt die Parallellektüre von Hunter Heycks bereits erwähntem Age of System nahe, dass es durchaus anonymere Kräfte waren, die undisziplinierte, „heterarchische“ Unternehmungen wie die Kybernetik zweiter Ordnung damals nach oben spülten (Abb. 1). Nach letzterer sucht man zwar vergeblich bei Heyck. Doch als Manifestation des Endes jenes Zeitalters, um welches sich Heycks Buch titelgebend dreht, fügt sie sich ziemlich genau ins Bild.

Was wäre also dieses Zeitalter, zu dessen post mortem-Symptomen man die Kybernetik zweiter Ordnung zählen könnte? Nun, es ist oder war eines, das sich im Wesentlichen durch eine Vielzahl komplexer und größtenteils funktionierender Systemzusammenhänge auszeichnete: immense Verwaltungsapparate, vertikal integrierte Industriekonzerne, massive Infrastrukturprojekte, starke Staaten und dergleichen mehr. „Industrialismus“, „industrialistische Ordnung“, „Industriekapitalismus“ und ähnliche Begrifflichkeiten kursierten hierfür seinerzeit (z.B. Gorz 1983). Die Unentrinnbarkeit solcher (eben) Systeme, und die Notwendigkeit diese auch irgendwie zu verwalten, beförderte nun, folgt man Heyck, nicht nur ein gewisses $\mathrm{Maß}$ an technokratischer Systemgläubigkeit, sondern auch ein ganz bestimmter Modus, über ,den Menschen' nachzudenken. In Heycks Diktion: „bureaucratic worldview“. Jener worldview wiederum entsprach eine ganz bestimmte oder jedenfalls dominante Form, Sozialwissenschaft zu betreiben - „high modern social science“ -, samt deren charakteristische Vorlieben für bestimmte Schlüsselbegriffe wie „Struktur“, „Funktion“, "Prozess“ oder „Modell“ (S. 23). Dazu zählte, so Heyck, neben operations research, Spieltheorie und Informationstheorie somit nicht zuletzt das, was man gerne als (wie auch immer) kybernetisch-inflektierte Humanwissenschaften der Nachkriegsjahrzehnte verbucht: Linguistik à la Noam Chomsky, Psychologie à la George Miller, Wirtschaftswissenschaften à la Herbert Simon. In der einen oder anderen Weise, so lautet Heycks zentrale These, 


\section{HIERARCHY}

A form of organization resembling a pyramid. Each level is subordinate to the one above it. [S.U.]

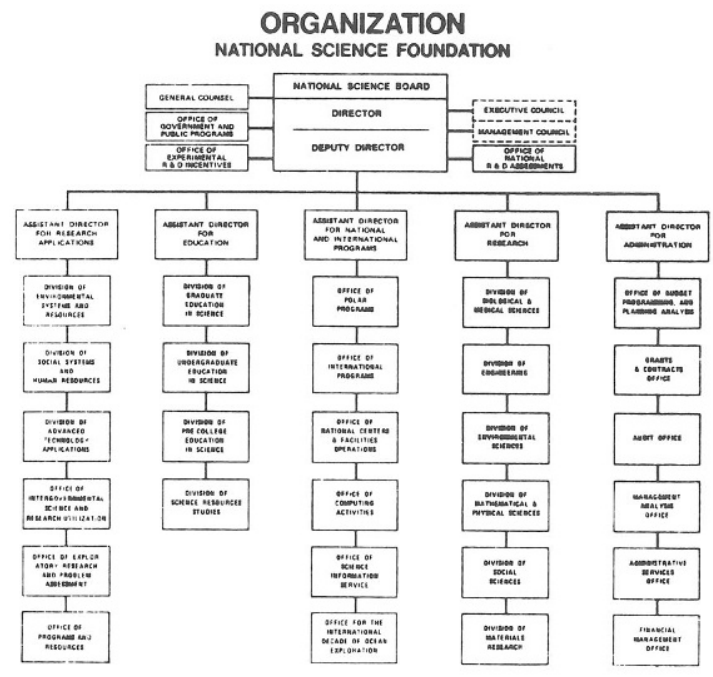

\section{HETERARCHY}

A form of organization resembling a network or fishnet. Authority is determined by knowledge and function. [S.U.]

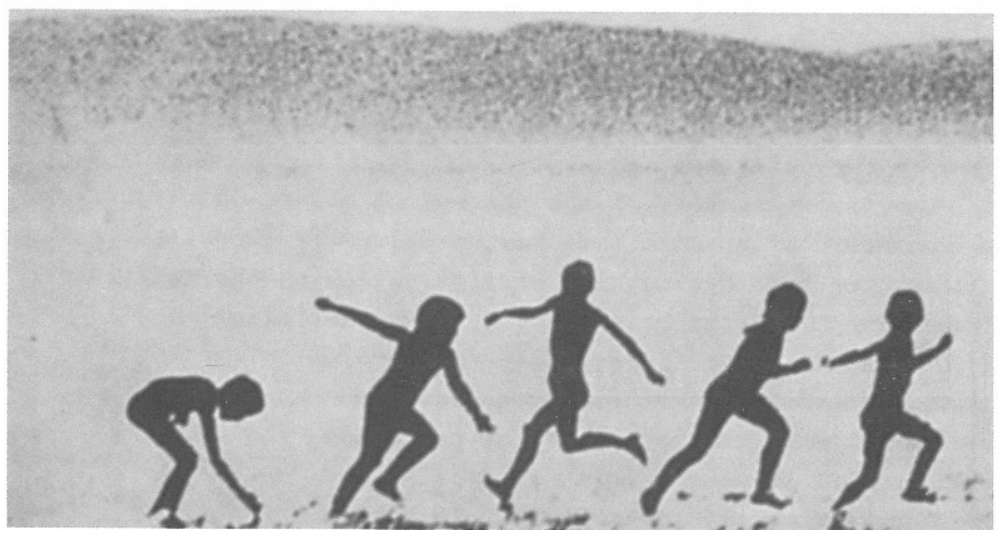

Abb. 1 Hierarchie und Alltag, ca. 1973/74. (Foerster 1995 [1974]: 64). Der Übergang zu "heterarchischen" Systemen bzw. zur Kybernetik zweiter Ordnung kam, so glaubte man am BCL, einem Paradigmenwechsel im Kuhn'schen sinne gleich 
waren all diese intellektuellen Projekte Symptome - und Architekten - der angedeuteten, industriell-bürokratischen Gemengelage.

Und das Ende dieses Systemzeitalters, das wäre also irgendwann in den 1970er Jahren zu suchen: ein Jahrzehnt, das bekanntlich ganz allgemein mit der Erosion etablierter Ordnungen einherging („nach dem Boom“, sagen Zeithistoriker hierzulande (Raphael \& Doering-Manteuffel 2008)). Dessen Anfang hingegen, so argumentiert Heyck, lässt sich ungefähr in den 1920er Jahren verorten. Womit der clue, wenn man so will, von Heycks historiographischem Synthesewerk eigentlich schon vorweggenommen ist. Dieser clue wäre: Der Versuch, in Anlehnung an den Unternehmenshistoriker Alfred D. Chandler Jr., für ein nicht unwesentliches Spektrum von wissenschaftlichen Unternehmungen, für die man reflexartig geneigt sein könnte, (Kalte) Kriegs-Logiken und entsprechende Periodisierungen in Anschlag zu bringen, ein anderes, eben im weitesten Sinne industriell-bürokratisches framing vorzuschlagen. Naturgemäß liest sich das stellenweise etwas schematisch (was auch an der quantitativen Unterfütterung der Ausführungen liegt). Und es trifft sich im Einzelnen durchaus mit dem, was man, wie Müggenburg, auch aus der biographischen Innenperspektive ableiten könnte. Heycks Thesen zur US-amerikanischen Wissenschaftspatronage der Nachkriegszeit (Kapitel 2) oder zum gleichzeitigen Anschwellen modellbasierter Wissensproduktion (Kapitel 6) etwa spiegeln sich ziemlich gut in Karrieren wie derjenigen Foersters. Heycks Anspruch, auf diesem Weg jedoch gerade das bigger picture zu readjustieren, ist dennoch (allermindestens) bedenkenswert, denn gewisse historiographische Selbstverständlichkeiten - die Computermetapher, die Rede von der Kriegsgeburt und dergleichen - werden damit zwar schon nicht ad acta gelegt, auf diesem Weg aber doch in deutlich abweichenden Dynamiken verortet. Das ist natürlich nicht ganz unerhört - man denkt hier etwa an die Verstrickungen von Informationstheorie und Telefonindustrie (Hagemeyer 1979; Mills 2011). Aber Heycks „new frame“ (S. 15) besticht, wenn auch nicht in jedem Detail, so doch in der Gesamtschau und einer gewissen, intuitiven Plausibilität. Selbst der vielbeschworene „Militärisch-Industrielle Komplex“ beinhaltet ja immerhin das Wörtchen „industriell“ - ein Umstand, der in der „Krieg ist der Vater aller Dinge“-Logik, wie sie etwa der neueren deutschen Medienwissenschaft in die Wiege gelegt wurde, tendenziell ausgeblendet wurde (z.B. Kittler 1984).

In Ansätzen wird so auch plausibel (auch das gehört zum Appeal des Buchs), wieso es mit jenem Typus hochmoderner Wissenschaft irgendwann wieder ein Ende finden sollte: insofern nämlich jene starren Systeme, deren Korrelat eine solche bürokratische Weltsicht gewesen sein mag, durch eine Reihe angeblich fluiderer Regimes verdrängt werden sollten. Sprich: „Märkte“, denen man nun zutraute, „collective rationality“ besser 
zu organisieren als die hierarchisch durchstrukturierten Behörden; oder der damit einhergehende Kult um unkonventionelle Unternehmer statt systemtreuer Experten (S. 203-204). Vor allem aber wirft Heycks BürokratieTopos ein neues - instruktives - Licht auf jene „Modelle“ des Menschen, welche die fraglichen Wissenschaften bekanntlich gerne fabrizierten. „The rational individual is, and must be, an organized and institutionalized individual", so hieß es etwa in Herbert Simons Frühwerk Administrative Behavior - ein Diktum, aus dem, so Heyck, weniger Systemantagonismus oder Cyborgträume sprachen, als vielmehr klassisch moderne Ängste (S. 127): die normative Haltlosigkeit der säkularen Welt einerseits, die Undurchschaubarkeit einer komplexen Gegenwart andererseits, die gar nicht anders als kollektiv bewältigt werden konnte. Daher das zeitgenössische Faible für Institutions-, Verwaltungs- und Organisationstheorien, die derartige Komplexität zu reduzieren hatten, was dann letztendlich soweit ging, derartig aufgedeckte Mechanismen mit den Mechanismen des Denkens selbst zu identifizieren. Letztendlich sei Psychologie „organization theory in miniature“, meinte etwa Simons zeitweiliger Kollege Allen Newell zu wissen (S. 139).

Dem Primat der notwendig organisierten Vernunft entsprach so ein Bild vom Menschen als ebenso notwendig begrenzte - „finite“ - Kreatur, ein Motiv, dem Heyck ebenfalls nachspürt: von der "limited channel capacity“ des menschlichen Wahrnehmungsapparats, mit der sich Kognitionspsychologen herumzuschlagen hatten (S. 86) bis hin zu jener „bounded rationality“, die Wirtschaftswissenschaftler damals für sich entdeckten (S. 92). Das scheint ziemlich weit weg vom Maschinenzauber Foerster'scher Provenienz; es gehört aber eigentlich nicht viel Phantasie dazu, sich auszumalen, wie ein solcher Blick (der bei Heyck ziemlich USA-zentriert daherkommt) auch für hiesigere Verhältnisse fruchtbar gemacht werden könnte. Von „Mängelwesen“ und „Institutionenbedürftigkeit“, von „Industriebürokratie“, „formaler Organisation“ und „verwalteter Welt“ war damals ja auch hierzulande einigermaßen systematisch die Rede.

\section{Systeme der Provinz}

Das wäre, zugegeben, aber eine andere Geschichte. Heycks Age of System überlappt sich dennoch weniger stark, als man das vielleicht erwarten könnte, mit der dritten System-Studie, die hier besprochen werden soll: Julian Bauers Zellen, Wellen, Systeme. Eine Genealogie systemischen Denkens, 1890-1980. Ein „entscheidender Fluchtpunkt“ dieser Genealogie, heißt es da, sei Luhmanns soziologische Systemtheorie (S. 5). Interessanterweise (es 
scheint am Thema zu liegen) bedient Bauer ebenfalls gleich eingangs das Zauberer/Lügner-Motiv, das, wie gesehen, auch Müggenburgs FoersterBuch durchzieht. Schon Otto Neurath nämlich wusste, dass der „Systematiker [...] ein geborener Lügner [ist], erfüllt von dem Drange, jenes Kunstwerk, das wir System nennen, vor die Augen des Betrachters zu zaubern“ (S. 1).

Die Gemeinsamkeiten brechen hier aber ab. Fungierte Foerster oben als eine Art Aussichtsturm auf die Systemlandschaften der Nachkriegszeit, versteht sich Zellen, Wellen, Systeme als mehr oder weniger protagonistenfreie Archäologie „systemischen Denkens“. Bauers erklärtes Ziel ist es dabei, dieses Denken zu „provinzialisieren“. Und zwar durch Rückführung auf dessen „heterodoxe“ Quellen, dessen „historisch tiefere[n] und eigenartigere[n] Wurzeln“ (S. 208). Dazu zählen, wie im weiteren Verlauf der Studie deutlich wird, so unterschiedliche Dinge wie die organismische Soziologie des 19. Jahrhunderts, die Parapsychologie, die theoretische Biologie, „periodische Geschichtsentwürfe“ im Stile Spenglers und die „frühe Wissenschaftsforschung" (z. B. Karl Mannheim). Was die orthodoxen und weniger eigenartigen Quellen dieses Denkens eigentlich wären, tritt dagegen weniger klar konturiert hervor. Nicht zu den heterodoxen und historisch tieferen Quellen gehört die Kybernetik, wie Bauer mit Verweis auf die bereits üppige Kybernetikliteratur wohl zurecht betont (S. 2) - die Kybernetik kommt dann entsprechend auch nicht mehr zur Sprache, wie überhaupt Bauers Genealogie 1890-1980 dazu neigt, vor allem im ersten Drittel des 20. Jahrhunderts herumzustöbern. Thesen der Art, wie Heyck sie in Age of System zusammengetragen hat, stoßen bei Bauer entsprechend auf wenig Gegenliebe (S. 26). Bauer interessiert sich zum Zweck der „Provinzialisierung“ (also durchaus legitim) für "tiefere“ Quellen solchen Denkens. Seinem Buch gelingt es allerdings nur bedingt, das Walten und Schalten dieser Quellen auf eine ähnlich griffige These zuzuspitzen.

Der Grundgedanke jener anderen Genealogie scheint zu sein, dass es, erstens, so etwas wie eine ,intellektuelle Kultur" systemischen Denkens gibt (S. 1); und dass es, zweitens, möglich ist, diese durch ein Set an Parametern einzugrenzen, die (vorwiegend) der neueren und teils nicht mehr ganz so neuen Wissenschaftsforschung entlehnt sind. Dazu gehören: „epistemic virtues“ (etwa Hang zur „Reflexivität“ oder „Paradoxie“), „Bildprogramme“ (Einsatz von Diagrammen, Tabellen und ähnlichem), „Grenzobjekte“ (hier: Modelle, Metaphern), „moral economies“, „Begriffsnetze“, „Zeitbögen“ und einiges mehr. Was sind das aber nun für Quellen und Denker (kaum Denkerinnen), die sich so zu Tage fördern lassen? Es sind, soviel sei gesagt Bauers Lesepensum verdient alle Achtung - ziemlich viele. Manche davon sind mehr (etwa: Karl Camillo Schneider, Alfred Vierkandt, Karl Blacher), 
manche weniger obskur (so etwa Uexküll, Bertalanffy, Spengler, Scheler). Und mehr oder weniger exakt erfüllen sie den skizzierten Kriterienkatalog.

Die Vielzahl von Akteuren, Lektüren und Vignetten, die Bauer mobilisiert, kann hier kaum wiedergegeben werden. Und tatsächlich fügt sich die Argumentation nur bedingt zu einem Ganzen. So leuchtet es zwar unmittelbar ein, dass etwa die „Parapsychologie“ (wie pseudowissenschaftliche Unternehmungen überhaupt) dazu neigte, Wissenschaftlichkeit, also auch Formen der Reflexivität, gewissermaßen überzusimulieren. Auch dass das Systemdenken von biologistisch-holistischen Versatzstücken durchsetzt ist, klingt plausibel. Oder: dass Ökonomen irgendwann in der Zwischenkriegszeit die "Wirtschaft" qua System und mittels Diagrammen, Zeitreihen und dergleichen erfanden. Wieso (und ob) diese und ähnliche Manöver aber miteinander kommunizierten, und in welcher Weise sie späteren Systemtheoretikern vom Schlage Luhmanns den Weg ebneten, bleibt - so mein Eindruck - offen. Formulierungen wie „stilbildend“ (S. 160), „präfigurieren“ (S. 199), „untergründig [...] orientierend“ (S. 211) häufen sich, bleiben aber eher suggestiv. Und nur ganz am Rande dringt in dieser Genealogie durch, auf welche Umstände und historischen Bedingungen diese Kulturen systemischen Denkens eigentlich jeweils reagierten (der Problemkomplex Nationalökonomie/Verkehrsinfrastrukturen prä-1914 scheint etwa dazu zu gehören).

Dass Luhmanns Sozialtheorie einige dieser Denker durchaus verarbeitet, jedenfalls zitiert, deutet sich in Zellen, Wellen, Systeme erst am Ende der Untersuchung an. Dazu zählt, so stellt sich heraus, etwa der belgische (und organismische) Sozialtheoretiker Guillaume de Greef (1842-1924): einer, der dem goldenen Zeitalter des Freihandels entstammte und bei dem gewisse Konzepte - wie "Heterarchie“ - also schon angelegt sein mochten (S. 292). Was das aber letztendlich bedeutet, wie die systemische Ideenkette genau funktioniert, hat sich, für diesen Leser jedenfalls, bei der Lektüre nicht so ganz erschlossen. Oder um den Meister des Zettelkastens selbst zu Wort kommen zu lassen: „Bestimmt das eigene Denken, wen man zitiert? Oder bestimmt der, den man zitiert, das eigene Denken?“ (Luhmann 1980).

Interessanter wäre dann womöglich doch die Frage, wieso derartige Konzepte - und dazu zählt nicht zuletzt das BCL-Vokabular: Autopoiesis, Selbstorganisation usw. - in den 1970er Jahren (und seither) dermaßen Karriere machen konnten. Wer sich für solche Fragen interessiert, oder überhaupt für die Kybernetik, die oder der wird Jan Müggenburgs Buch mit Gewinn lesen; Hunter Heycks Buch wird sie oder ihn diesbezüglich mit historiographischen Grundsatzfragen konfrontieren; Julian Bauers Buch dagegen wird vielleicht eher diejenigen Leser*innen überzeugen, die sich für die abstrakteren Dimensionen der Ideengeschichte interessieren. 
Am Grundimpetus, nämlich das Andenken an Systemtheorie oder Kybernetik zu „provinzialisieren“, ist sicher nichts auszusetzen; tatsächlich wäre dies zeitgemäß. Die eingangs angerissene Alternative - Aufklärung oder Silicon Valley - scheint in jedem Fall an der Sache vorbeizugehen. Allein schon deswegen (auch das lässt sich diesen drei Büchern je auf ihre Weise entnehmen), weil die Kybernetik bestenfalls ein Puzzlestein in dieser Geschichte wäre.

\section{Anmerkungen}

1 Damals waren es etwa der Haraway-Schüler Paul Edwards oder, hierzulande, friedensbewegte Informatiker und angehende Medienwissenschaftler, die der frühen Kybernetik zu spätem Ruhm verhalfen. Siehe unter anderem Edwards (1986) sowie Bickenbach et al. (1985).

2 In der akademischen Variante findet sich diese Gegenerzählung prominent etwa bei Andrew Pickering (2010).

3 Siehe etwa Theodore Roszak (1986); die aktuelle Version ist düsterer, siehe etwa Fred Turner (2019).

4 „Konferenz: Aktualisierung Kybernetischen Denkens,“ 2018, https://www.facebook. com/events/113203699472542/.

5 Siehe etwa Marie Hicks (2017), David Gugerli (2018), Frank Bösch (2018); zu einem ähnlichen Ergebnis kommt man, macht man sich z. B. die Mühe, dem iPhone historisch nachzudenken, siehe Mariana Mazzucato (2018).

6 Einen Überblick über von Foersters Tätigkeiten gibt das online-Findbuch des Heinzvon-Foerster-Archivs: http://www.univie.ac.at/heinz-von-foerster-archive/.

7 Zum Topos „reliability“ siehe weiterführend Edward Jones-Imhotep (2017).

\section{Literatur}

Bickenbach, Joachim, Reinhard Keil-Slawik, Michael Löwe und Rudolf Wilhelm (Hg.) 1985. Militarisierte Informatik. Schriftenreihe Wissenschaft und Frieden. Marburg: BdWi.

Bösch, Frank (Hg.) 2018, Wege in die digitale Gesellschaft. Computernutzung in der Bundesrepublik 1955-1990. Göttingen: Wallstein.

Edwards, Paul 1986. Border Wars: The Science, Technology, and Politics of Artificial Intelligence. Radical America (19): 39-50.

Edwards, Paul 1988. The Closed World: Computers and the Politics of Discourse. Dissertation, University of California.

Foerster, Heinz von 1948. Das Gedächtnis. Eine quantenphysikalische Untersuchung. Wien: Deuticke.

Foerster, Heinz von 1979. Cybernetics of Cybernetics. In: Klaus Krippendorf (Hg.). Communication and Control in Society. New York: Gordon and Breach: 5-8.

Foerster, Heinz von (Hg.) 1995 [1974]: Cybernetics of Cybernetics: Or, the Control of Control and the Communication of Communication. Minneapolis, MN: Future Systems, Inc.

Galison, Peter 1994. The Ontology of the Enemy: Norbert Wiener and the Cybernetic Vision. Critical Inquiry (21): 228-66.

Gorz, André 1983. Wege ins Paradies. Thesen zur Krise, Automation und Zukunft der Arbeit. Berlin: Rotbuch Verlag. 
Groebner, Valentin 2008. Das Mittelalter hört nicht auf. Über historisches Erzählen. München: C.H. Beck.

Gugerli, David 2018. Wie die Welt in den Computer kam. Frankfurt/Main: Fischer.

Hagemeyer, Friedrich-Wilhelm 1979. Die Entstehung von Informationskonzepten in der Nachrichtentechnik. Eine Fallstudie zur Theoriebildung in der Industrie- und Kriegsforschung. Dissertation, FU Berlin.

Heidenreich, Stefan Heidenreich und Markus Krajewski 2001. Das erste Bit ist gelöscht. Ein Nachruf auf den Kommunikationstheoretiker Claude Shannon. de:bug. Elektronische Lebensaspekte (46): 32 .

Hicks, Marie 2017. Programmed Inequality: How Britain Discarded Women Technologists and Lost Its Edge in Computing. Cambridge/MA: MIT Press.

Hombach, Dieter (Hg.) 1982. Zeta 01. Zukunft als Gegenwart. Berlin: Rotation Verlag.

Jones-Imhotep, Edward 2017. The Unreliable Nation. Hostile Nature and Technological Failure in the Cold War. Cambridge/MA: MIT Press.

Kittler, Friedrich 1984. Alan Turing und die moderne Kriegsmaschinerie. Das Gespenst im Computer. Überblick (8): 46-47.

Kittler, Friedrich 1994. Der zerstreute Mathematiker. Norbert Wiener zum 100. Geburtstag. Frankfurter Allgemeine Zeitung (26. Nov.): 4 (Sonntagsbeilage).

Kline, Ronald 2015. The Cybernetics Moment: Or Why We Call Our Age the Information Age. Baltimore: Johns Hopkins University Press.

Luhmann, Niklas 1980. Ideengeschichte in soziologischer Perspektive. In: Joachim Matthes (Hg.). Lebenswelt und soziale Probleme: Verhandlungen des 20. Deutschen Soziologentages zu Bremen 1980. Frankfurt/Main: Campus: 49-61.

Mazzucato, Mariana 2018. The Entrepreneurial State: Debunking Public vs. Private Sector Myths. London: Penguin Books.

Mills, Mara 2011. Deafening: Noise and the Engineering of Communication in the Telephone System. Grey Room (43): 118-143.

Müller, Albert und Karl H. Müller (Hg.) 2007. An Unfinished Revolution? Heinz von Foerster and the Biological Computer Laboratory | BCL 1958-1967. Wien: echoraum.

Paul, Joachim 2018. Offener Brief an Richard David Precht. vordenker.de (blog) (26. April 26), http://www.vordenker.de/blog/?p=1997 (22.12.2018).

Pickering, Andrew 2010. The Cybernetic Brain: Sketches of Another Future. Chicago: University of Chicago Press.

Raphael, Lutz und Anselm Doering-Manteuffel 2008. Nach dem Boom: Perspektiven auf die Zeitgeschichte seit 1970. Göttingen: Vandenhoeck \& Ruprecht.

Rid, Thomas 2016. Maschinendämmerung. Eine kurze Geschichte der Kybernetik. Berlin: Propyläen Verlag.

Roszak, Theodore 1986. From Satori to Silicon Valley: San Francisco and the American Counterculture. San Francisco: Don't Call It Frisco Press.

Steinbuch, Karl 1969. ,Zwei Kulturen': Ein engagierter Beitrag. In: Helmut Kreuzer (Hg.). Literarische und naturwissenschaftliche Intelligenz. Dialog über die „zwei Kulturen“. Stuttgart: Klett: $143-54$.

Turner Fred 2019. Machine Politics. The Rise of the Internet and a New Age of Authoritarianism. Harper's Magazine (January 2019), https://harpers.org/archive/2019/01/ machine-politics-facebook-political-polarization/ (22.12.2018).

Vester, Frederic 1978. Unsere Welt, ein vernetztes System. Stuttgart: Klett-Cotta. 
Max Stadler

Professur für Wissenschaftsforschung

ETH Zürich

Clausiusstraße 59

8092 Zürich

Schweiz

stadler@collegium.ethz.ch 